\title{
Hybrid Ray/Wave Optics for Laser-Plasma Interaction
}

R.P. Ratowsky, J.S. Kallman, B.B. Afeyan, M.D. Feit

February 18, 1999

U.S. Department of Energy

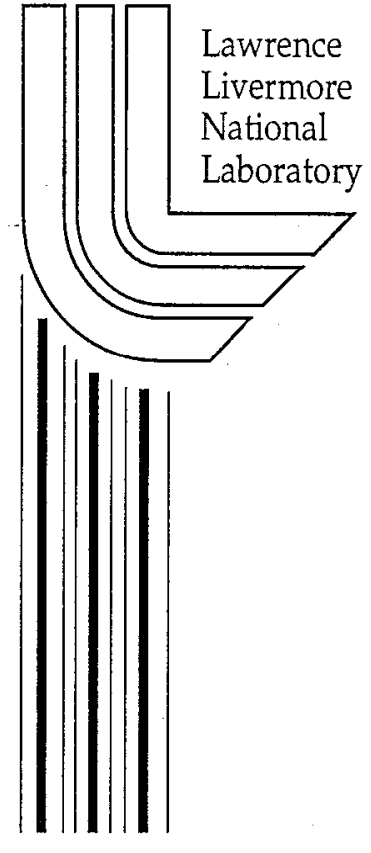




\section{DISCLAIMER}

This document was prepared as an account of work sponsored by an agency of the United States Government. Neither the United States Government nor the University of California nor any of their employees, makes any warranty, express or implied, or assumes any legal liability or responsibility for the accuracy, completeness, or usefulness of any information, apparatus, product, or process disclosed, or represents that its use would not infringe privately owned rights. Reference herein to any specific commercial product, process, or service by trade name, trademark, manufacturer, or otherwise, does not necessarily constitute or imply its endorsement, recommendation, or favoring by the United States Government or the University of California. The views and opinions of authors expressed herein do not necessarily state or reflect those of the United States Government or the University of California, and shall not be used for advertising or product endorsement purposes.

This report has been reproduced directly from the best available copy.

Available to DOE and DOE contractors from the Office of Scientific and Technical Information

P.O. Box 62, Oak Ridge, TN 37831

Prices available from (615) 576-8401, FTS 626-8401

Available to the public from the

National Technical Information Service

U.S. Department of Commerce

5285 Port Royal Rd.,

Springfield, VA 22161 


\title{
Hybrid Ray/Wave Optics for Laser-Plasma Interaction
}

\author{
Richard P. Ratowsky, Jeffrey S:Kallman, Electronics Engineering/DSED \\ Bedros B. Afeyan, Polymath Associates \\ Michael D. Feit, Lasers
}

\begin{abstract}
This aim of this FY 1998 LDRD project was to create a computational tool which bridges the gap between wave and ray optical regimes, important for application areas such as laser propagation in plasma and multimode photonics. We used phase space methods, where a set of rays distributed in a particular way in position and angle retain many essential features of wave optics. To characterize and enhance our understanding of the method, we developed a GUI-based photonics tool which can analyze light propagation in systems with a variety of axial and transverse refractive index distributions.
\end{abstract}

\section{INTRODUCTION}

Until the mid-19th century, natural philosophers argued vigorously over whether light was in reality a wave or a particle. Compelling reasons existed for both points of view: light seemed to travel mostly in straight lines like a particle, but showed diffraction and interference like a wave. In the early 20 th century the same issues surfaced again in the context of the quantum theory, where both light and matter exhibited both wave-like and particle-like behavior, depending on the observational setting.

The ability of light to act like a wave or a particle has practical consequences for the calculation of its propagation. For systems large compared to a wavelength, such as conventional bulk optics, particle-like geometrical optics is usually a good approximation. On the other hand, if the scale of features is on the order of a wavelength, such as at a focal point, solving a wave equation is necessary to capture the physics of diffraction. Often, as in this bulk optics example, both circumstances arise in subdomains of the same problem.

Many practical situations important to LLNL programs share this dual length-scale property. One important application area is laser propagation in fusion plasmas. Traditional modeling uses ray tracing to transport the laser intensity and this is usually a good approximation. But when plasma gradients are wavelength scale, as may occur in regions of NIF targets, a wave optical treatment is essential. Another relevant area is multimode photonics. A multimode optical fiber may be optically large, but calculating the effects of interference between the modes-speckle-is of critical importance.

The purpose of our FY98 LDRD proposal, "Hybrid Ray/Wave Optics for LaserPlasma Interaction" was to create a computational tool which would move easily between the wave and ray optical regimes. We accomplished this by using phase space methods, where a set of rays distributed in a particular way in position and angle retain many essential features of wave propagation, including diffraction ${ }^{1}$. By launching the right set of rays, diffraction can be calculated directly from the ray distribution without explicitly solving a wave equation. In this way, a problem domain can easily use both ray and wave optics in the regions where the descriptions are most appropriate. To characterize and enhance our understanding of the method, we developed a GUI-based photonics tool which can analyze light propagation in systems with a variety of axial and transverse refractive index distributions.

This article will give an overview of our work in FY 1998. First, we will convey the principles of the phase space method and give some simple examples. Then, we will describe the software tool we developed to study the propagation of fields through the Wigner method. This tool allows us to study the important issue of the appropriate sampling of rays in the phase space to achieve desired accuracy. Further examples of light 
propagation in systems with a variety of axial and transverse refractive index distributions will be given. Finally, we will describe some of the limitations of our current scheme and the path we envision for further development and applications.

\section{PROGRESS}

The Method. We usually think of an electric field as a vector quantity which varies in space and time according to Maxwell's electromagnetic field equations. We can also describe the field in terms of the wavenumbers or spatial frequencies which comprise it ("Fourier representation"). However, it is sometimes most natural to think in terms of a mixed representation, whereby the field is thought of as a set of spatial frequencies, the spectrum of which changes with position. This is analogous to the way music, which is a pressure oscillation changing in time, can be represented by a musical score which shows a set of pitches (frequencies) changing in time. For the electric field, a wavenumber defines a direction in space; the coordinates of the direction are a set of angles. Thus the field can be represented by a function of position and angle, which defines the ray phase space.

One such function is known as the Wigner distribution, introduced by E. P. Wigner in $1923^{2}$. The Wigner distribution allows quantum mechanical quantities to be calculated using a distribution function on a classical-like position-momentum phase space, much as we will use it to calculate wave optical properties on a position-angle phase space. Mathematically the Wigner transform can be thought of as a Fourier transform not of the field, but of its correlation function relating the field at two points in space. An example of a Wigner distribution is shown in Fig. 1. for a Gaussian field and for a uniformly illuminated aperture. Qualitatively, the reason diffraction is included in the ray description is that at each point in space one has a fan of ray angles: this is just a manifestation of the Fourier uncertainty principle. The Wigner distribution is not strictly a phase space density, however, since it is not always positive.
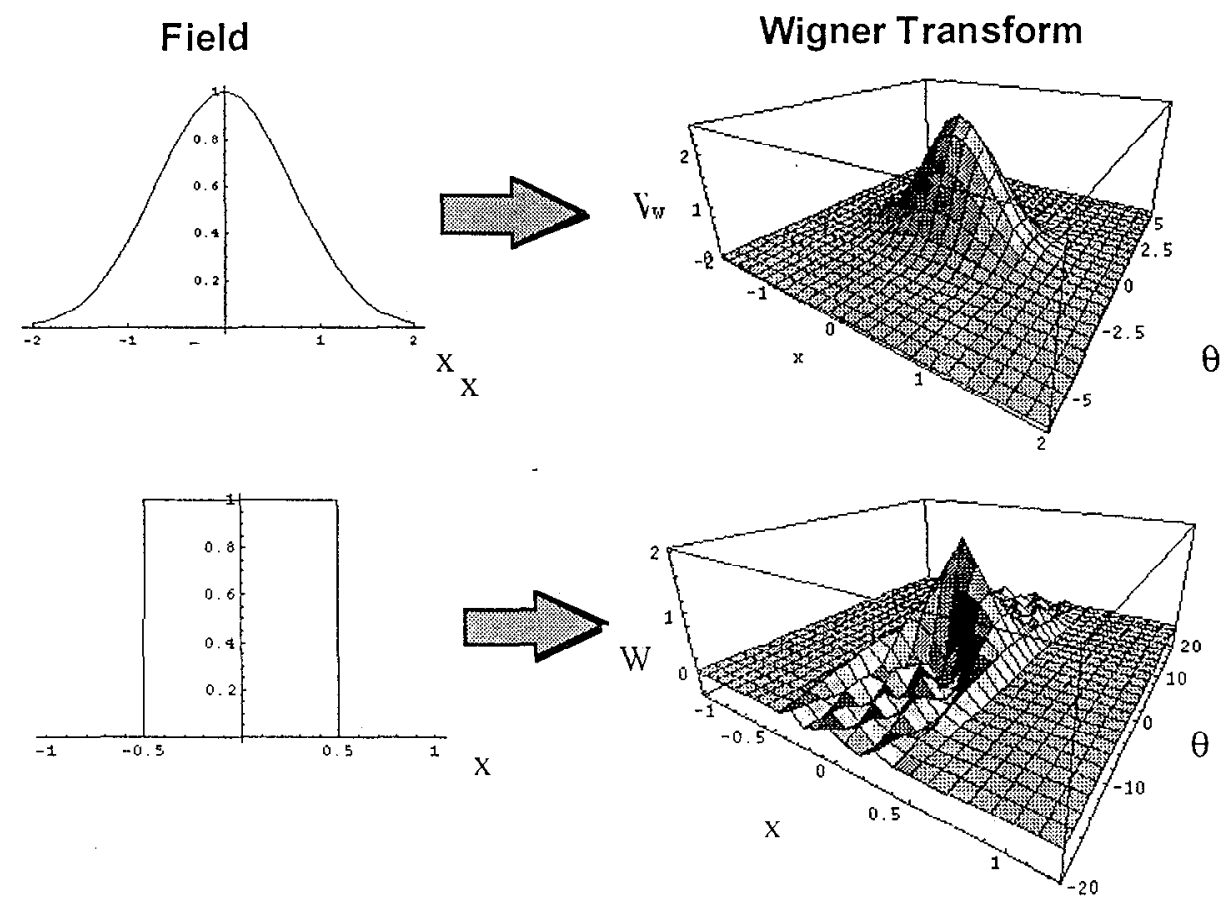

Figure 1. Wigner phase space distributions for a Gaussian field (top) and a uniformly illuminated aperturc (bottom). 
The Wigner propagation algorithm works as follows. From an initial complex electric field distribution, we calculate the Wigner transform. This distribution is then evolved in space by simply transporting it along rays, that is, assuming conservation along a ray. This will be an excellent approximation if the index is not too rapidly varying on a wavelength scale. If at every position in the ray phase space for the new (evolved) distribution, we integrate over all ray angles, we obtain the intensity distribution ("nearfield") as a function of spatial position. Conversely, if at each angle in phase space we integrate over position, we obtain the intensity distribution as a function of ray angle ("farfield"). Since in wave tics the nearfield and farfield amplitudes are related by Fourier transform, the complex electric field can be reconstructed (up to a constant phase factor) from the ray intensity distributions in position and angle.

As an example of a calculation using the Wigner propagation algorithm, we calculated the diffraction pattern from a double slit (see Fig. 2). The Wigner function is calculated in the plane of the slits, then propagated along rays to the plane of the screen. The calculated result and the exact solution are overlaid, and agreement is excellent. In fact, for free propagation, the Wigner method is formally exact, and the only errors are due to sampling. This illustration shows strikingly that diffraction and interference, usually considered outside the domain of ray optics, can be obtained through ray tracing the Wigner distribution.

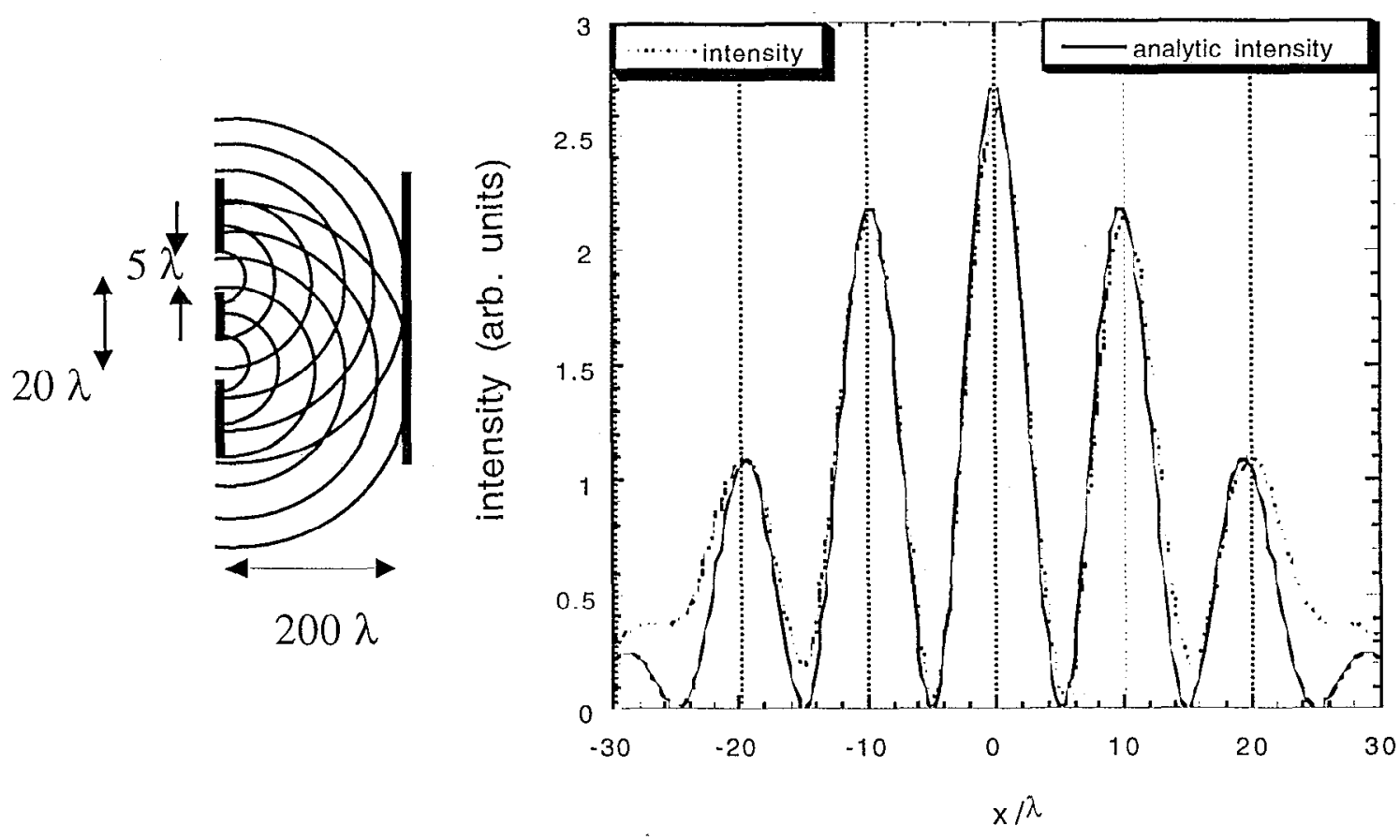

Figure 2. Wigner phase space method calculation of double-slit diffraction by ray tracing.

PHASTER. To study the Wigner method we developed a GUI-based code which allows us to propagate beams in a variety of media in two spatial dimensions. We call the code "PHASTER": Phase Space Techniques for Electromagnetics Research. PHASTER allows us to set up an arbitrary initial beam consisting of a sum of Gaussians with selected widths and amplitudes. After computing and displaying the Wigner distribution for the beam, it will solve the ray equations for a set of points in the ray phase space which sample the Wigner distribution in a prescribed manner. The rays are traced using an adaptive Runge-Kutta method through a variety of refractive index distributions having both transverse and axial variation. After propagating the prescribed set of rays to the exit plane, 
it will display the phase space distribution at the exit plane, as well as the $\mathrm{x}$-space and angle space intensities (nearfield and farfield). PHASTER gives useful insight into the dynamics of the rays in phase space and their effect on the wave optical distribution.

Our first example of a PHASTER calculation is shown in Fig. 3. A Gaussian beam is shown propagating through three "soft slabs", i.e., small regions where the refractive index rises and falls, depicted in the central window of the GUI. The contour plot on the left shows the initial Wigner distribution of the Gaussian. Below it we see the X-space intensity, and to the left the angle-space intensity (farfield). The windows on the far right show the analogous data for the propagated distribution. Note that the phase space distribution has become elongated and tilted: this is simply a graphic depiction of diffraction. Rays at large magnitude angles (top and bottom of distribution) move fastest in $\mathrm{x}$, while small angle rays (near center of distribution) move little. The result is a spreading of the $x$-space distribution while the angular distribution does not change, exactly what we would expect from simple free-space diffraction. Additional spreading in $\mathrm{x}$-space occurs due to the plates, but not in angle because rays receive no transverse impulse from the axially varying index.

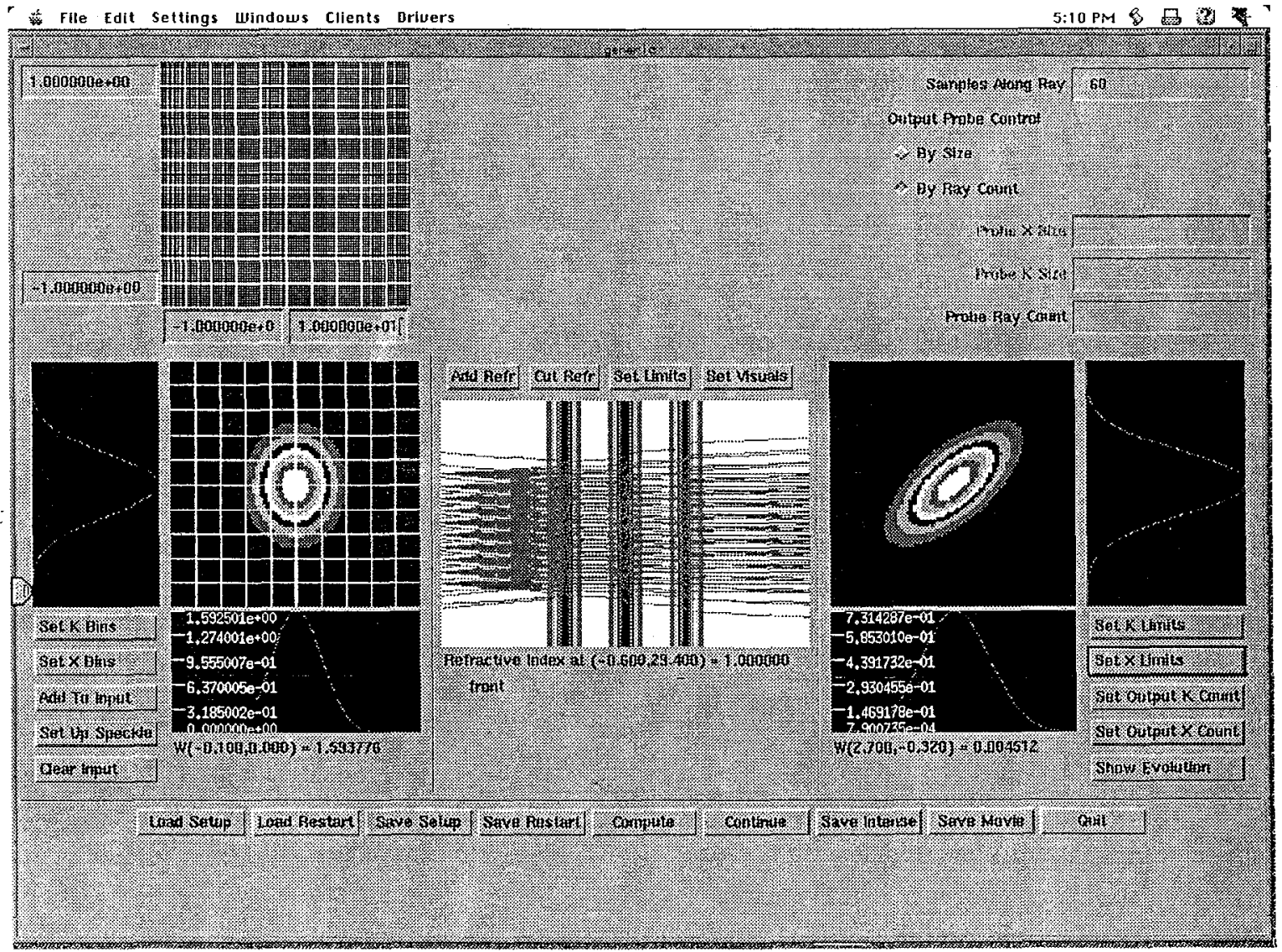

Figure 3. PHASTER calculation of propagation through three slabs. The tilted distribution on the right is the manifestation of diffraction in the ray phase space. 
Fig. 4 shows propagation in a waveguiding structure. The rays are obviously confined to the waveguide, and at the exit have formed a 'galaxy' shaped distribution. A given ray will encircle the origin as it traces out a periodic path, but the frequency of the rotation decreases as the angle increases, causing the spiraling.

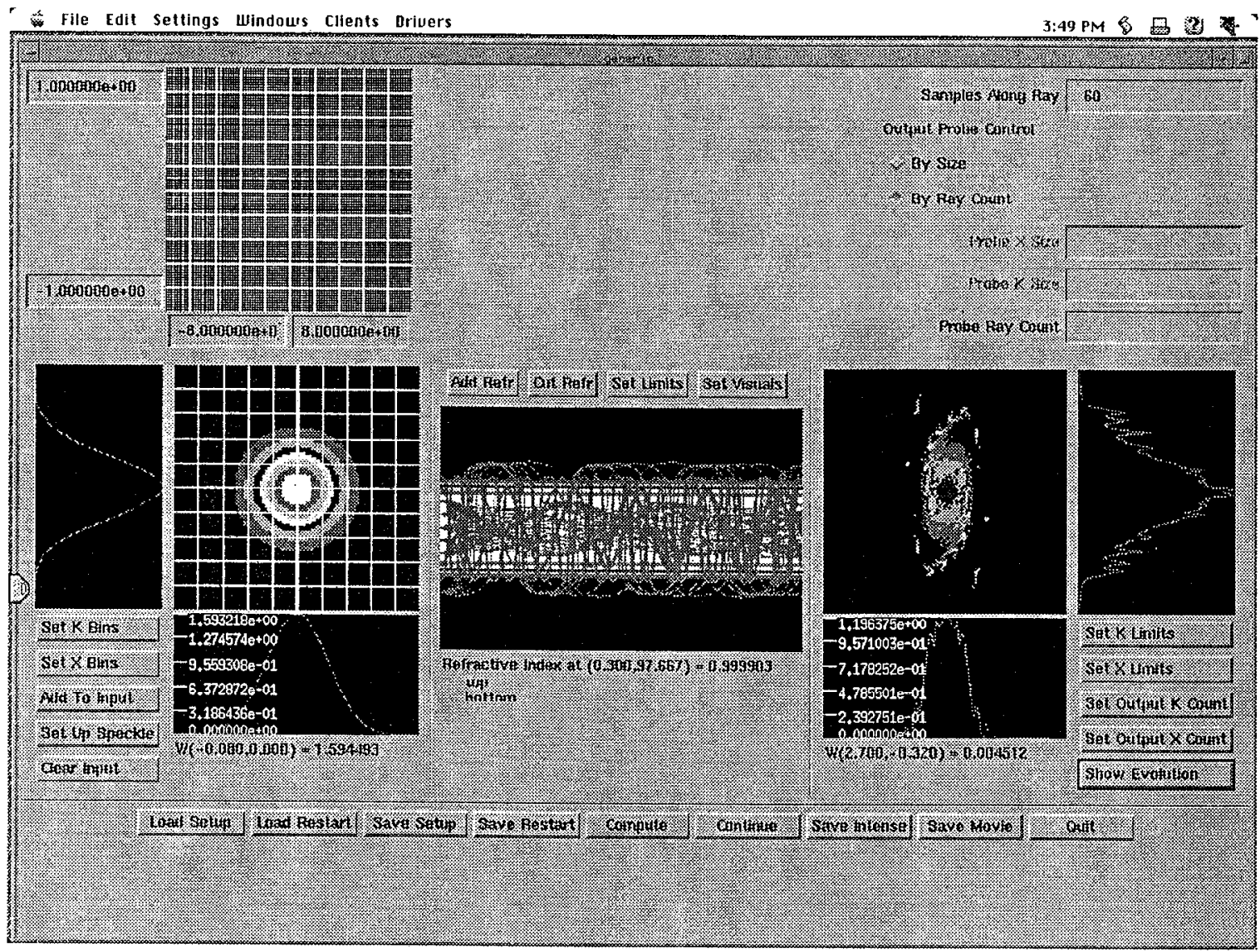

Figure 4. PHASTER calculation of waveguide propagation, showing rays with transverse turning points. 
A third example is shown in Fig. 5. Here a beam is incident at an oblique angle on a linear index gradienl, a model for a laser propagating in a plasma. A subset of the rays is reflected by the plasma index gradient, while the rays getting through form the distorted distribution on the right. This example is of particular interest because the problem admits an exact solution in terms of Airy functions ${ }^{3}$ and thus will allow direct evaluation of accuracy.



Figure 5. PHASTER calculation for oblique incidence on an inhomogeneous plasma with a linear density gradient. 
Our final example, Fig. 6, shows the propagation of a speckled beam through the inhomogeneous plasma. By speckled we mean that we are launching a set of Gaussians with a distribution of transverse tilt angles and random phases. Propagation of speckle is important for laser-plasma interaction physics and also for propagation in multimode fiber. Again an exact solution to this problem is available.

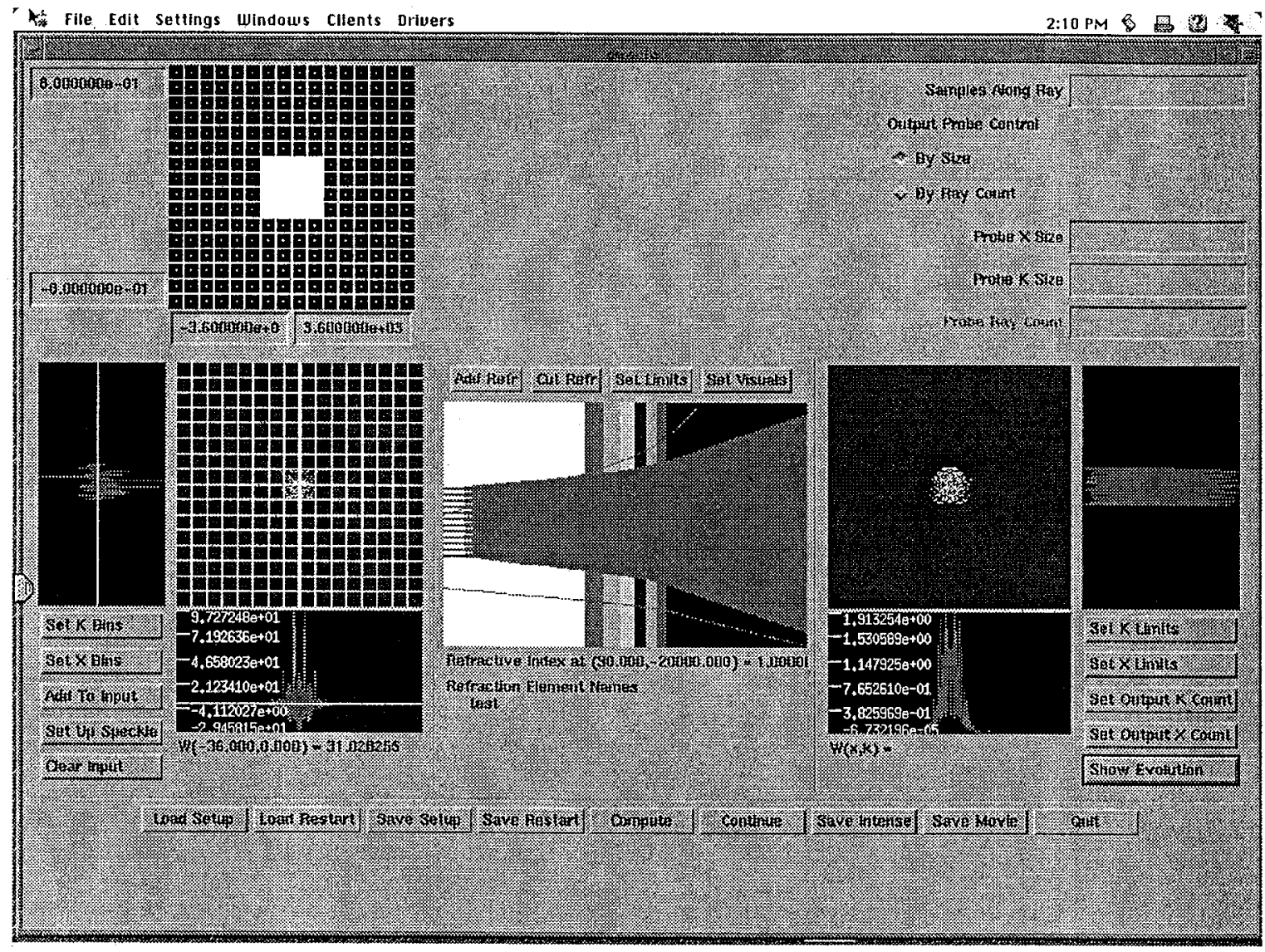

Figure 6. PHASTER calculation for speckled beam at normal incidence on inhomogeneous plasma

An important feature of the code is its flexibility in sampling the phase space at the entrance plane so as to put a higher density of rays where the Wigner distribution has larger values. Sampling is critical for the method, because if we do not sample wisely we suffer in computational efficiency for a given accuracy. PHASTER allows us to divide the phase space into rectangular bins and vary the size of the subdivisions of the bins.

\section{FUTURE WORK}

The Wigner method shows great promise as at wave propagation algorithm, and much remains to be done to fully exploit its capabilities. To treat reflections accurately, for example, requires extending the calculation to the time domain. The method will be most effective when it is relatively inexpensive to trace rays, as in bulk optics where all that is required is Snell's law refraction (and reflections if wanted) at boundaries; we are in the process of developing a three-dimensional bulk optics solver. We have also heretofore ignored polarization, which can also be included. More subtle effects such as accurately calculating tunneling will require propagating complex rays. 
For the method to come to full fruition, we have recognized that using rays is only a first step. Because rays are one-dimensional, it is difficult to sample a two or threedimensional space effectively. One extension is to use "fat rays" or Gaussian basis functions whose center propagates along a ray. Ultimately we envision using a wavelet basis, which can optimally sample a system which has disparate length scales. Resolution can be applied only where needed and crude representations retained where they cause minimal harm. This way, from rays To full wave optics in phase space can be simulated by retaining a hierarchy of successively more accurate approximations.

In FY 1999, we are focusing on applications of the Wigner method to multimode photonics, where effective simulation tools are in demand but full wave optics solvers are computationally difficult ${ }^{4}$.

${ }^{1}$ E. Wolf, "Coherence and Radiometry,", J. Opt. Soc. Am. 68, 6-17 (1978); M. Bastiaans, "Application of the Wigner distribution function in optics", in The Wigner Distribution: Theory and Applications to Signal Processing, ed. W.F.G. Mecklenbräuker, North-Holland (1997).

${ }^{2}$ E. Wigner, "On the quantum correction for thermodynamic equilibrium", Phys. Rev. 40, 749-752 (1979).

${ }^{3}$ B. B. Afeyan, Bull. Am. Phys. Soc. 41, No.7, paper 9Q2, p. 1598 (1996).

${ }^{4}$ R. P. Ratowsky, B. B. Afeyan, J. S. Kallman and M. D. Feit, "Propagation modeling for multimode photonics", UCRL-JC-131253-ABS, 1998 